\title{
MAGNETIC RECONNECTION AS THE ORIGIN OF GALACTIC RIDGE X-RAY EMISSION
}

$2 D$ Numerical Simulation of Reconnection driven by Parker Instability and Supernova

\section{S. TANUMA}

Department of Astronomy, School of Science, University of Tokyo

7-3-1 Hongo, Bunkyo-ku, Tokyo 113, Japan

T. YOKOYAMA, T.KUDOH AND K. SHIBATA

National Astronomical Observatory of Japan

2-21-1 Osawa, Mitaka-shi, Tokyo 181, Japan

R. MATSUMOTO

Department of Physics, Faculty of Science, Chiba University

\section{AND}

K. MAKISHIMA

Department of Physics, School of Science, University of Tokyo133 Yayoi-cho, Inage-ku, Chiba 263, Japan

7-3-1 Hongo, Bunkyo-ku, Tokyo 113, Japan

We present a scenario for the origin of the hot plasma in our Galaxy, as a model of a strong X-ray emission $\left(L_{\mathrm{X}}(2-10 \mathrm{keV}) \sim 10^{38} \mathrm{erg} \mathrm{s}^{-1}\right)$, called Galactic Ridge X-ray Emission (GRXE), which has been observed near the Galactic plane. GRXE is thermal emission from hot component $(\sim 7 \mathrm{keV})$ and cool component $(\sim 0.8 \mathrm{keV})$. Observations suggest that the hot component is diffuse, and is not escaping away freely. Both what heats the hot component and what confines it in the Galactic ridge are still remained puzzling, while the cool component is believed to be made by supernovae. We propose a new scenario: the hot component of GRXE plasma is heated by magnetic reconnection, and confined in the helical magnetic field produced by magnetic reconnection or in the current sheet and magnetic field. We solved also the 2-dimensional magnetohydrodynamic (MHD) equations numerically to study how the magnetic reconnection creates hot plasmas and magnetic islands (helical tubes), and how the magnetic islands confine the hot plasmas in Galaxy. We conclude that the magnetic reconnection is 

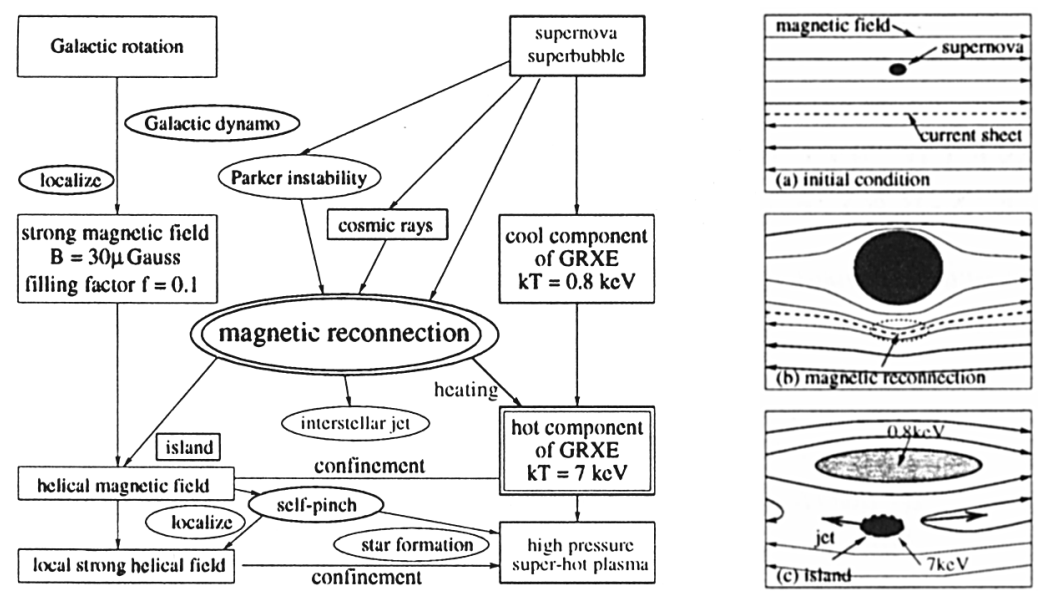

Figure 1. The schematic diagram showing possible routes for the Galactic Ridge X-ray Emission.

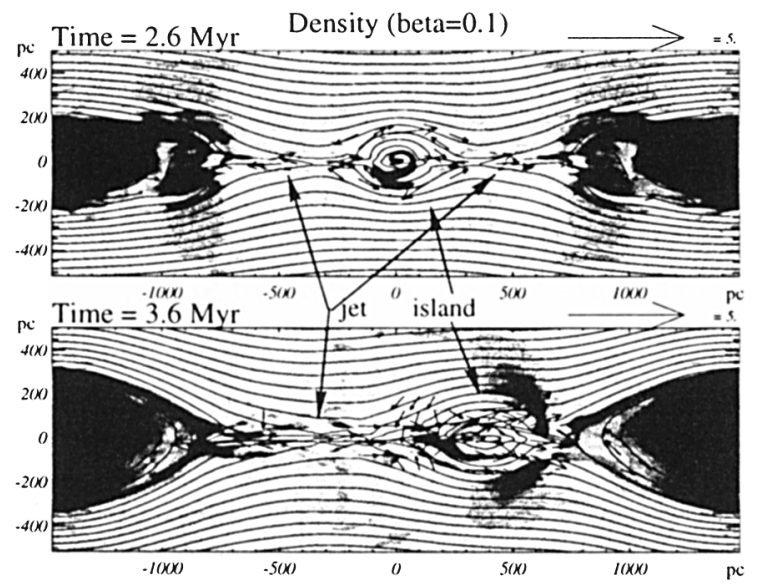

Figure 2. 2D numerical simulation of magnetic reconnection Driven by Supernova.

able to heat up the cool component to hot component of GRXE plasma if the magnetic field is localized into intense flux tube with $B_{\text {local }} \sim 30 \mu \mathrm{G}$ (the volume filling factor of $f \sim 0.1$ ).

\section{References}

Kaneda, H., Makishima, K., Yamauchi, S., Matsuzaki, K. \& Yamasaki, N., (1997), ApJ, in press $(11 / 1)$.

Tanuma, S., Yokomama, T., Kudoh, T., Matsumoto, R., Shibata, K. \& Makishima, K. (1992), ApJ, submitted

Yokoyama, T. \& Shibata, K., (1995), Nature, 375, 42 